\title{
Letter to the Editor on the Article: "Mixed Reality Anatomy Using Microsoft HoloLens and Cadaveric Dissection: a Comparative Effectiveness Study"
}

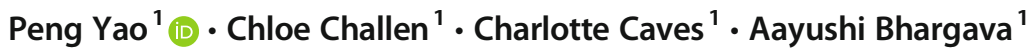 \\ Published online: 16 December 2019 \\ (C) International Association of Medical Science Educators 2019
}

Dear Editor,

The role of immersive digital learning in anatomy education was recently explored in Medical Science Educator by Stojanovska and colleagues [1]. The authors compared anatomy examination scores from students taught on mixed reality (MR) devices with those taught using traditional cadaveric dissection. Although they claim that exam performance was similar regardless of study modality, we believe that there are a few possible improvements that may serve to better investigate the effectiveness of MR as an educational tool.

The authors infer that "medical students, regardless of study modality, performed similarly." However, we would like to emphasize that results between cohorts using different modalities cannot be compared if a global average is taken, which includes the scores of all those cohorts and modalities. Instead, we would suggest that students with upper limb anatomy MR training have their scores on upper limb questions compared against the rest of the participants, and the students with lower limb MR training similarly compared with other student performances on lower limb questions. Equally, any graphs should distinguish data from MR and control groups.

Finally, in understanding the utility of MR in anatomy education, we would like to encourage the supplementation of results with some qualitative data. For example, does MR lend itself to more self-study time and periodic revision of the material? This might explain why the authors found that the MR device seemed to be more efficient for teaching anatomy than traditional cadaveric dissection. Exploration of student experience, engagement, and motivations for learning are critical to better understanding of the most effective aspects of the MR environment as a learning platform.

\section{Compliance with Ethical Standards}

Conflict of Interest The authors declare that they have no conflict of interest.

Ethical Approval NA

Informed Consent NA

\section{Reference}

1. Stojanovska M, Tingle G, Tan L, Ulrey L, Simonson-Shick S, Mlakar J, et al. Mixed reality anatomy using Microsoft HoloLens and cadaveric dissection: a comparative effectiveness study. Med Sci Educ. 2019:1-6. https://doi.org/10.1007/s40670-019-00834-x.

Publisher's Note Springer Nature remains neutral with regard to jurisdictional claims in published maps and institutional affiliations.
Peng Yao

peng.yao15@imperial.ac.uk

Imperial College London, London, UK 